\title{
Sachregister zu Band 82
}

Die fett gedruckten Zahlen bedeuten Eigenarbeiten. Bb. = Buchbesprechungen.

Ablatio retinae 8911., 222, 254,

343

hämorrhagische - durch Ader-hautblutung 73

Bedeutung des Glaskörpers bei Entstehung der - 343

Acarusmilben in den Meibom-

schen Drüsen 213. Adaptation 310 Akkommodationslähmung,

angeborene 242 Aktinomyces discofoliatus in

den Tränenröhrchen 214 Albinismus 237 Alkoholinjektionen, orbitale

286 Anatomie, normale und patho-

logische - 213 (Bericht) Angiom der Chorioidea 75

- des Gehirns in Beziehung zumSehorgan 175

Angiomatosis retinae 351

- und des Gehirns 96Angiopathia retinae trauma-

tica 76 Angiospasmus der Netzhautge-

fäße 84. Aniridie 236 Anophthalmus 233 Arachnodaktylie und Linsen-

luxation 241 Area striata, Form und Ausdeh-

nung der - 147 Argyll-Robertsonsches Phä-

nomen 150 Argyrol, Silberablagerung in der

Hornhaut nach Einträufelung

von - 330 Arsenpräparate, Schädigungen

des Sehnerven durch - 142 Arteria centralis retinae, Ver-

schluß der $-84 \bullet-$ Druckerhöhung der - nach

Commotio cerebri 84, 160 Arteria hyaloidea persistens

239-Aubert-Förstersches Phäno-

men 319 Auge, Modellierung des lebenden

- 342 Augenbecherblatt, Entwicklung

des äußeren - 228 Augendominanz 321 Augenermüdung 321

Augenf arbenmutationen,

durch Röntgenbestrahlung her-

vorgerufen 231 Augenhintergrund, Verände-

rungen des - bei Blutkrank-

heiten 15

- Veränderungen des - bei Sy-ringomyelie 325

- bei Nachtblindheit 177 Augenlider s. Lider Augenmuskeln, angeborene Ano-

malien der - 242 Augenwinkel, Anomalie der Stel-

lung des äußeren - 213

B

Bacillus anthracoides als Ur- 
sache einer Panophthalmie 339 Bacillus subtilis, Ringabszeß

mit Panophthalmie durch den

- 217 Barraquers Augenklinik 353 (Bb.) Begutachtung, ärztliche -in der

Krankenversorgung no (Bb.) Beleuchtung und Sehschärfe 313 Bildnis und Spiegelbild 353 (Bb.)

Bindehaut s. Conjunctiva Blickkrämpfe bei postenezpha-

litischem Parkinsonismus 154 Blindgeborene, Raum- und Ge-

staltsauffassung bei operierten

- vor und nach der Operation

109 (Bb.) Blindheit, Ursachen der - von

1000 Fallen 348 Blutkrankheiten, Augenhinter-

grundsveränderungen bei -15

Augenstörungen bei -160

Netzhautveränderungen bei -

85 Bluttränen 160 Brille, Geschichte der - 251 Bulbusabschnitt, Untersuchun-

gen des vorderen $\mathbf{\square}-\cdot$ mit dem

Fluoreszenzmikroskop 269 Bulbusfistel, Setzung einer hin-

teren -331

C Chiasma 144

Chorioidea, Pathologie der - 73 (Bericht)

- $\quad$ isolierte Gliaherde der - 75, 218

- $\quad$ Krebsmetastasen in der - 340Chorioideagefäße, Rolle der -

in der Pathogenese 337

Sachregister zu Band 82.

355

Chorioiditis disseminata, tu-

berkulöse 218 Chorioiditis guttata 76 Chorioretinitis juxtapapilla-

ris 77 Coats sche Erkrankung 78, 97 Commotio cerebri, Überdruck

in den Netzhautarterien als

Spätfolge von - 84, 160 Conjunctiva, Erkrankungen der

$-\quad 215$

- $\quad$ Tuberkulose der - 254Conjunctivalschürze 234Conjunctivitis pseudomem-

branacea, Ätiologie der - 328 Conjunctivitis rosacea, Rönt-

genbehandlung bei -344 Cornea, Entwicklung der $\cdot-226$

- $\quad$ Erkrankungen der - 216

feinst punktierte Veränderungen der -123

C. farinata 123

Mißbildungen der -236

eigenartige Degeneration beider

$-\quad 323$

- $\quad$ kristallinische Entartung der -

325

- $\quad$ weiße Ringein der - 106, 107

- $\quad$ chronisches Ödem der - 255Cysticercus, subretinaler 80

$\mathrm{D}$

Dermoide, Entstehung der - 243 Diabetes mellitus, Refraktions-veränderungen bei 
- $\quad$ Netzhautveränderungen bei -82

Dominanz der Augen 321 Doppelbilder, Entstehung von - nach äußeren Radikalopera-tionen der Stirnhöhlen 172 Druck in den Netzhautgefäßen 80,

81 - und intrakranieller Druck 155 Durahämatom 158 Dystrophia adiposa epithelialis

lentis 220 Dystrophia adiposogenitalis infolge von Lues congenita 149

E Eidetische Fähigkeiten 317 Einschlußkörper, Züchtung der

- in Gewebskultur 345 Eklampsie, Retinopathie bei -

83 Elektrische Hochspannung,

paralyseähnliches Krankheits-

bild nach Berühren einer - 160 Elektrokoagulation bei post-

operativer Iriszyste 166

- $\quad$ bei Netzhautabíösung 93Embolie, konstitutionelle Disposition zur - 348

Encephalitis epidemica, Au-gensymptome nach - 154

Encephalomyelitis disseminata und Augenkrankheiten 152

Endokrine Drüsen, Einfluß der

- $\quad$ auf das Vestibularorgan 71Entwicklungsgeschichte des

Auges 226 (Bericht) Epibulbäre Tumoren 3 Epicanthus 247 Epilepsie, Augenhintergrundsver-

änderungen bei -159

- $\quad$ spastische Ischämie der Netz-haut im epil. An fall 84

Ermüdung der Augen 321 Exkavation, tiefste randständige - - ohne Glaukom 349

$\Gamma$

Farben, Wirkung von - 318 Farbensinn 306 Feuersta rablösung 219 Filaria in der Netzhaut 80.

Flocculus in der Vorderkammer

237 Fluoreszenzmikroskop, Unter-

suchungen des vorderen Bulbus-

abschnittes mit dem - 269 Fokalinfektion, Chorioiditis

durch -73

- $\quad$ Netzhautschädigungen durch -

77 Formwahrnehmungen 319 Fovea, Aplasie der - 97 Fremdkörper, interessante Lage

eines - in der Bindehaut 338 Frühj ahrskatarrh, Behandlung

des -345 Fuchsscher Fleck 88

$\mathrm{G}$

Gauchersche Krankheit, Veränderungen der Chorioidea bei

- $\quad$ 75Gedächtnis, optisches 317Gehirnsk'erose, tuberose 96Gehirnabszeß

158Gehirndiagnostik, Bedeutung

des Nystagmus für die - 72, 148 Gehirntumoren, Augensym-

ptome bei - 156, 157 Geschichte der Augenheilkunde

248 (Bericht) Gesichtsfeldprüfungen 309 Gestaltauffassung bei operier-ten Blindgeborenen vor und nach der Operation 109 (Bb.) Gestaltswahrnehmungen 319 Glaskörper, Bedeutung des bei Entstehung der Ablatio retinae 343 Glaskörperabhebung, ringför-mig abgerissene - 166

$23^{*}$

356

Sachregister zu Band 82.

GlaskÖrperblutung, rezidivie-

rende 78 Glaukom 220 
Wesed und Ursprung des pri-mären - 255

und Hydrophthalmus 232

- - und Naevus flammeus 231

- $\quad$ akutes - mit Zyklodialyse ope-riert 339

Gliagewebe in der Chorioidea 75,

218 Glioma retinae 96 Grippe, Nasennebenhöhlenkatarrh

infolge von - mit letalem Aus-

gang 338

- $\quad$ Retinitis bei - 77Größenwahrnehmungen 319Gunnsches Phänomen 243

$\mathrm{H}$

Haftgläser 354 (Bb.) Hämangiom der Netzhaut 96 Hamartom, epibulbäres - bei

Hydrophthalmus 175 Haradasche Krankheit 74 Hemeralopie, angeborene 311 Hemianopsie, homonyme 148

- $\quad$ einseitige - bei Sehnervenatro-phie 143

Hemikephalie 244

Hepato-lenticuläre Degeneration, Augenerscheinungen bei - 159

Heredodegeneration der Macula 88

Heterochromie der Iris 237

- des Sympathicus 180, 238Hippelsche Krankheit 221Hippus bei multipler Sklerose

153Hornerscher Muskel 229Hornhaut s. CorneaHydrophthalmus 232, 233

- epibulbäres Hamartom bei -

175 Hypertonie am Augenhinter-grund 82

- $\quad$ Netzhautgefäßveränderungenbei - 168

Hypophysis, Tumoren der -

145. 156 Hypopyoniritis, rezidivierende

218 Hypotonie, Erzeugung akuter -

am Kaninchenauge 326

I

Idiotie, familiäre amaurotische 87

- $\quad$ ohne Maculabefund 188Ignipunktur bei Netzhautab-

lösung 91 Iktero-hämorrhagische Spiro-chätose, Augensymptome bei der - 255

Indicia 256 (Bb.) Infektionen, Augenerkrankun-

gen nach - 154 Intravasaler Druckin den Netz-

hautgefäßen und intrakranieller

Druck 80 Iris, Entwicklung der - 227

Aufsplitterung des vorderen Stromablattes der - 218

Vererbung sektorenförmiger Pig-mentierung der - 239

Iriszyste, postoperative - durch Elektrokoagulation geheilt 166

$\mathrm{J}$

Japaner, Anatomie des Auges der

Kammerbucht, Anatomie der -

217 Kammerwasser, postmortaleVer-

änderungen des - 347 Kampimeter 309 Karzinommetastasen in der

Chorioidea 340 Katarakt, angeborene 240 Kataraktoperation, intrakap-

suläre - mit basaler Iridek-

tomie 338 
- $\quad$ Korrektion fehlerhaft geheil-ter staroperierter Augen 342

Keilbeinrücken, Meningiom des

- 352 Keratitis nummularis 167 Keratitis parenchymatosa 216

e lue congenita 176

Luetinreaktion bei -346

- und Sattelnase 12Keratitis rosacea, Röntgenbe-

handlung bei - 344 Keratoconjunctivitis sicca

215 Knochenbildung im Auge 139 Knochenbrüchigkeit, abnorme

- und blaue Sklera 234 Kolobom 234

- $\quad$ des Sehnerveneintritts beimHuhn 230

Kontaktgläser, neue 342 Kontusionsspätrosetten der

Linse 220 Kupferkatarakt 220

$\mathrm{L}$

Lateralsklerose, amyotrophi-

sche $\cdot-$ mit nystagmusartigen

Zuckungen 159 Laurence-Biedlsches Syn-

drom 87 Leitungsbahnen, anatomischer

Aufbau der optischen - 146 Lenticonus posterior, Entsteh-

ung des -241

Sachregister zu Band 82.

357

Leukämie, Augenveränderungen bei - 219

- $\quad$ Erkrankungen der Chorioideabei -- 75

Leukoplakie der Bindehaut 215

Lichtsinn 299

Li der, Entwicklung der $\cdot-228$

- $\quad$ Erkrankungen der - 213

- $\quad$ Melanose der - 239Lidreflex 321

Linse, angeborene Anomalien der

- 240 - Erkrankungen der - 219, 220

- $\quad$ Pigmentierung der Hinterflächeder - nicht entzündlichen Ur-sprungs 330

Linsenkapsel, Anatomie der - 219

Lipämie der Netzhaut bei Diabetes 83

Lipoidose der Conjunctiva 322

Lochbildungin der Macula 76, 87

Lochbrille, Ergänzung zur ·-匹 191

Luetinreaktion bei Keratitispar-enchymatosa 346

Lupus erythematosus, Augen-komplikationen bei -78

Luxatio bulbi posttraumatica 211

Lymphoblastische Wucherun-gen des Auges 218

Lymphozytengeschwulst an Lid und Bindehaut, mit Rönt-genstrahlen behandelt 340

$\mathrm{M}$

Macula, traumatisches Loch der

- $\quad$ mit Schädigung einer hinte-ren Ciliararterie 326

Malaria, Netzhautschädigungen 
bei - 77 Mai en, wie malten sich die alten

Meister selbst ? 353 (Bb.) Mangan-Thyroxin, Behandlung

der tabischen Sehnervenatro-

phie mit - 340 Masern, Affektion der Macula bei

- 77

Medikamente, Netzhautschädigungen durch - 77 Megalocornea 235

- $\quad$ und Hydrophthalmus 232Meibomsche Drüsen, Pseudo-

tumoren der - 214 Melanosarkom der Papule 174 Melanose der Bindehaut, bösartig

entartet 339

- $\quad$ der Lider und der Sklera 239Membrana pupillaris persis-

tens 239 Meningiom der Olfactoriusrinne

257 - des Keilbeinrückens 352

Meningitis tumorosa 108 Menstruation, Netzhautblutun-

gen bei der - 85 Metalues, Veränderungen des Seh-

nerven bei - 142 Migräne, Netzhautveränderungen

bei - 84 -- Oculomotoriuslähmung bei -

159 Mikrophthalmus 233, 234 - anatomische Untersuchung ei-

nes - 223 Mißbildungen des Auges 230

(Bericht) Modellierung des lebenden Auges

342 Mongolismus 244 Morton-Spiegel, zu einem Com-

bergschen Ophthalmoskop um-

gebaut 334 Musculus ciliaris, Entwicklung

des - 229 Musculus lacrimalis 229 Myasthenia gravis pseudo-

paralytica, Bewegungslosig-

keit der Bulbi bei - 159

N Nachbilder 304 Nachtblindheit, Augenhinter-

grund bei - 177 Naevus flammeus und Glaukom

231 Nebenhöhlen, Beziehungen der

-- zu den Erkrankungen des

Sehnerven 223 Nebenhöhlenerkrankungen

und Neuritis retrobulbaris 141 - infolge Grippe mit letalem Aus-

gang 338 Nervenkrankheiten und Auge

149 (Bericht) Nervus opticus, Anatomie des -

223

- $\quad$ Pathologie des - 141 (Bericht) - -atrophie, tabische 150

-- - tabische - mit Mangan-Thyroxin behandelt 340 Netzhaut s. Retina Neuritis retrobulbaris

141,142 Neurofibrillitis 77 Nierenleiden, Retinopathie bei

- 83 Nystagmographie 65 Nystagmus 64 (Bericht)

der Neugeborenen 67

der Bergarbeiter 66, 112 (Bb.)

willkürlicher 339

- Bedeutung des optomotorischen - für die topische Hirndiagno-stik 72,148 I $\mathbf{a}$ - bei Tabes

71,151

358

Sachregister zu Band 82. 
Oguchische Krankheit 86 Olfactoriusrinne, Meningiom

der - 257 Operationszwang bei Unfallge-

schädigten 1 Ophthalmia sympathica 220

bei Aderhautsarkom 74

Netzhautveränderungen bei $-\mathbf{\square} 79$

Ophthalmoskop, zu einem Com-bergschen - umgebauter Morton-Spiegel 334

Optik, physiologische 299 (Bericht)

- $\quad$ psychologische 317 (Bericht)-- Geschichte der - 251Optokinetischer Nystagmus

68 Orbitaltumoren 194, 223

$\mathrm{P}$

Panophthalmie, verursacht

durch Bacillus anthracoides 339 Pantocain 134 Papilla nervi optici. Entfär-

bung der - $105-\cdot$ Melanosarkom der - 174 Paralyse und Syphilis 149 Parkinsonismusund

Syphilis 149 Periarteritis nodosa 79

Augenveränderungen bei -218

Beteiligung der Chorioidea bei

- $\quad$ 74Perimetrie 309Periphlebitis retinae 78Pflege der Augen 353 (Bb.)Phakomatosen

96Photochemische Prozesse 302Physiologische Optik 299 (Bericht)

Piezometer 127 Pigmentdegeneration der Netz-

haut 86, 87, 221 Pigmentierung, gruppenförmige

- $\quad$ der Retina 97, 239Pigmentproben zur Prüfung von

Farbensehen 346 Pigmentstreifenbildung und

Pseudoxanthoma elasticum 222 Pigmentstreifenerkrankung,

angioide - des Fundus 88 Pilzflora des Auges 341, 342 Pneumokokkenstämme, Bio-

logie der - 217 Poliomyelitis, Augensymptome

bei -155

Pseudoencephalitis der Alko-holiker, Augenmuskellähmungen bei - 158

Pseudoxanthoma elasticum und Pigmentstreifenbildung 222

Psychologische Optik 317 (Bericht)

Ptosisope ration nach Blasko-vics, Erfolge der - 342

Pupillarmembran, Entwicklung der - 227

Pupillotonie mit Fehlen von Sehnenreflexen 159

$\mathrm{R}$

Rachitis und Cataracta zonularis

240 Radium als schädigendes und hei-

lendes Mittel 350 Raumauff assung bei operierten

Blindgeborenen vor und nach

der Operation 109 (Bb.) Raumsinn 311 Raumwahrnehmungen 319 Retikulo-Endothelialsystem

224 Retina, Anatomie der - 221 •- Pathologie der - 76 (Bericht)

- $\quad$ Rolle der Gefäße der - in derPathogenese 337

-- Blutungen der - 85

gruppenförmige Pigmentierung der - 97, 239

Risse der $-89,91$

- - Genese der Risse der - 254 Retinitis albuminurica 83 Retinitis centralis 80 Retinitis exsudativa Coats 78 , 
97 Retinitis hypertonica 81 Retinitis punctata albescens

87 Retinitis juxtapapillaris 107 Retinitis proliferans 78 Retinitis pseudoalbuminuri-

ca 78 Retinitis stellata 84 Röntgenbestrahlung, Katarakt

durch -220

S Sarkom der Chorioidea 75

- $\quad$ angioplastisches - in der Ader-haut und den Meningen 345

Sattelnase, Keratitis parenchy-

matosa und - 12 Schädelbildung, Anomalien der

- 243 Schauanfälle bei postenzephali-

tischem Parkinsonismus 154 Schüller-Christiansche

Krankheit 350 Schwangerschaftsniere, Reti-

nopathie bei - $\mathbf{- 8 3}$, 84 Schwangerschaftsunterbre-

chung und Netzhautablösung

94 Sehbahnen, Anatomie der - 223 I - Pathologie der --141 (Bericht)

Sachregister zu Band $82 . \quad 359$

Sehschärfe 311

Sinus cavernosus, Thrombophlebitis des - 224 Sklera, blaue 234

Kalkherde der --217

Melanose der - 239

senile Entartung der - an den Ansatzstellen der geraden Au-genmuskeln 113

- $\quad$ senile Verdünnung der - 164Sklerose, multiple, und Augen-

krankheiten 152 - - und Neuritis retrobulbaris 141 Sklerotomie, modifizierte hintere

$-\quad 331$

Skotom, zentrales 143 Spirochaeta myelophthorai53 Spirochätose, Augensymptome bei der

iktero-hämorrhagischen

- 255Sprengverletzungmitbeginnen-

der Meningitis, operativ geheilt 165 Stauungspapille, Ursachen der

$-\quad 143$

- $\quad$ bei Syringobulbie 155Stirnhöhle, Entstehung von Dop-

pelbildern nach äußeren Radi-

kaloperationen der -172 Strahlenschädigung der Retina

76 Streuungstuberkulose, häma-

togene 224 Subretinale Flüssigkeit 90 Sympathicusheterochromie

180, 238 Synästhesie 317 Syphilis und Auge i49ff.

- Chorioretinitís bei - 73Syphilisreaktionen 345Syringobulbie, Stauungspapille

bei - 155 Syringomyelie, Fundusverände-rungen bei -325

$\mathrm{T}$

Tabes, Nystagmus bei -- 71, 151 Tarsus, Entwicklung des - 228 Teratome, Entstehung der - 243

Thorium-X 344 Thrombose, konstitutionelle Disposition zur - 348 Tiefensehen 314 Tiere,

Beobachtungen an -320 Tonoskopie 80 Tortuositas vasorum 85, 96 Trachom, Pathogenese des

$-277$

Bakteriologie des -215

Tränenabflußwege bei - 346 Trachom, Verteilung der -

Kranken in Budapest 347

Tränen, blutige 160 Tränenabflußwege bei Trachom

346 Tränenröhrchenblennorrhoe 
der -214

- $\quad$ Pilzkonkremente in den - 214Tränenwege, Anomalien der -

242 Trichinose, Netzhautarterienver-

engerung bei - 84 Tuberkulose, hämatogene Streu-

ungs- -224

Chorioretinitis bei -73

der Netzhaut 77 Tuberose Hirnsklerose 96 Tumor en, epibulbäre 3

intraorbitale 31

der Chorioidea 75, 76 Tunica vasculosa lentis, Rück-

bildung der -228

U Unfallgeschädigte, Operations-zwang bei - - 1

$\mathrm{V}$

Vena centralis retinae, Ver-

schluß der - 85 Vestibulärer Nystagmus 70 Vorderkammer, Entwicklung der

- 227, 229

- $\quad$ Epithelauskleidung der - 217Vorstellungen 317

W

Weiße Ringe in der Hornhaut

106, 107 Wortreiz, PupiUenreaktion durch

$-\quad 321$

$\mathrm{X}$

Xanthomatose, Veränderungen der Chorioidea bei - 75

- $\quad$ Netzhautveränderungen bei -

87 Xerose der Bindehaut und Hornhaut 324

$\mathrm{Z}$

Zentralnervensystem, Nystagmus bei Erkrankungen des - 71

Zerebrospinalflüssigkeit bei Lues und Metalues 149

Ziliarkörper, gutartige Epithel-wucherung des - 218

Zyklodialyse. Abänderung der--

334

-. - $\quad$ bei akutem Glaukom 339Zyklopie 233

Zystoide Degeneration der Netzhaut 89 